\title{
On dihedrants admitting arc-regular group actions
}

\author{
István Kovács • Dragan Marušič • \\ Mikhail E. Muzychuk
}

Received: 26 September 2009 / Accepted: 9 August 2010 / Published online: 4 September 2010

(C) Springer Science+Business Media, LLC 2010

\begin{abstract}
We consider Cayley graphs $\Gamma$ over dihedral groups, dihedrants for short, which admit an automorphism group $G$ acting regularly on the arc set of $\Gamma$. We prove that, if $D_{2 n} \leq G \leq \operatorname{Aut}(\Gamma)$ is a regular dihedral subgroup of $G$ of order $2 n$ such that any of its index 2 cyclic subgroups is core-free in $G$, then $\Gamma$ belongs to the family of graphs of the form $\left(K_{n_{1}} \otimes \cdots \otimes K_{n_{\ell}}\right)\left[K_{m}^{\mathrm{c}}\right]$, where $2 n=n_{1} \cdots n_{\ell} m$, and the numbers $n_{i}$ are pairwise coprime. Applications to 1-regular dihedrants and Cayley maps on dihedral groups are also given.
\end{abstract}

Keywords Arc-transitive graph · Cayley graph · Cayley map · Dihedral group · Core-free group

\section{Introduction}

All groups in this paper are finite, and all (undirected) graphs and digraphs are finite and simple. For a (di)graph $\Gamma$, let $V(\Gamma), D(\Gamma)$, and $\operatorname{Aut}(\Gamma)$ denote the vertex set, the arc set, and the group of all automorphisms of $\Gamma$, respectively. For a graph $\Gamma$, let $E(\Gamma)$ denote the edge set of $\Gamma$; then two vertices $u$ and $v$ of $\Gamma$ are said to be

The research of István Kovács and Dragan Marušič was supported in part by "Agencija za raziskovalno dejavnost Republike Slovenije," research program P1-0285.

I. Kovács · D. Marušič $(\bowtie)$

FAMNIT, University of Primorska, Glagoljaška 8, 6000 Koper, Slovenia

e-mail:dragan.marusic@upr.si

D. Marušič

IMFM, University of Ljubljana, Jadranska 19, 1000 Ljubljana, Slovenia

M.E. Muzychuk

Department of Computer Science and Mathematics, Netanya Academic College, University st. 1, 42365 Netanya, Israel 
adjacent if $\{u, v\}$ is in $E(\Gamma)$. For a digraph $\Gamma$, a vertex $u$ of $\Gamma$ is said to be adjacent to another vertex $v$ of $\Gamma$ if $(u, v)$ is in $D(\Gamma)$. A $k$-arc of a (di)graph $\Gamma$ is a sequence of $k+1$ vertices $v_{1}, v_{2}, \ldots, v_{k+1}$ in $V(\Gamma)$, not necessarily all distinct, such that any two consecutive terms are adjacent and any three consecutive terms are distinct. For a subgroup $G \leq \operatorname{Aut}(\Gamma)$, we say that $\Gamma$ is $G$-k-arc-transitive, $G$ - $k$-regular if $G$ acts transitively, regularly, respectively, on the set of $k$-arcs of $\Gamma$. In particular, Aut $(\Gamma)$ $k$-arc-transitive and Aut $(\Gamma)-k$-regular are usually referred to as $k$-arc-transitive and $k$-regular, respectively. Let $G$ be a group with identity element $e$, and $S \subset G$ with $e \notin S$. The Cayley digraph $\operatorname{Cay}(G, S)$ over $G$ relative to $S$ is the digraph with vertex set $G$, and $(x, y) \in G \times G$ is an arc if and only if $x y^{-1} \in S$. Note that, when $S=S^{-1}$ is inverse closed, then $(x, y)$ is an $\operatorname{arc}$ of $\operatorname{Cay}(G, S)$ if and only if so is $(y, x)$; hence Cay $(G, S)$ can be also regarded as a graph, in which case the terminology Cayley graph is used.

The question which Cayley digraphs $\operatorname{Cay}(G, S)$ are $s$-arc-transitive for some $s>0$ has been studied extensively. For instance, all 2-arc-transitive digraphs $\operatorname{Cay}(G, S)$ are classified when $G$ is an abelian group, see [14], or a dihedral group, see [4, 15, 16]. As for 1-arc-transitivity, arc-transitivity for short, a complete classification is known over cyclic groups, see [8, 12], or can be found implicitly in [18, 19].

A natural next step to consider is the class of connected, arc-transitive Cayley graphs over dihedral groups, that is, the class of arc-transitive dihedrants. Notice that it is not restrictive to consider only Cayley graphs instead of Cayley digraphs. Namely, if Cay $\left(D_{2 n}, S\right)$ is connected and arc-transitive, where $D_{2 n}$ is a dihedral group of order $2 n$, then $S=S^{-1}$ must hold, and hence Cay $\left(D_{2 n}, S\right)$ is in fact a Cayley graph. In this paper we focus on a the subclass of dihedrants $\Gamma$ that admit a subgroup of automorphisms $G$ acting regularly on the arc set $D(\Gamma)$, or shortly, an arc-regular subgroup $G \leq \operatorname{Aut}(\Gamma)$. In particular, if $G=\operatorname{Aut}(\Gamma)$, then $\Gamma$ is called 1-regular. Such graphs have been studied in a number of papers: dihedrants of valency 4 or 6 in $[10,22,23]$, those of prime valency in [7], and finally, a construction of a 1-regular dihedrant of valency $2 k \geq 4$ is given in [11].

Here we consider the family $\mathcal{F}$ of dihedrants $\Gamma$ satisfying the following condition:

(A) $\Gamma$ is a connected dihedrant over the dihedral group $D_{2 n}$ which admits an arcregular action of a subgroup $D_{2 n} \leq G \leq \operatorname{Aut}(\Gamma)$, and moreover, any cyclic group of index 2 in $D_{2 n}$ is core-free in $G$.

Recall that, for a group $A$ and its subgroup $B \leq A$, the core of $B$ in $A$ is the largest normal subgroup of $A$ contained in $B$, denoted by $\operatorname{core}_{A}(B)$.

For graphs $\Gamma_{1}$ and $\Gamma_{2}$, the lexicographical product $\Gamma_{1}\left[\Gamma_{2}\right]$ is the graph with vertex set $V\left(\Gamma_{1}\right) \times V\left(\Gamma_{2}\right)$, and $\left(u_{1}, u_{2}\right)$ is adjacent to $\left(v_{1}, v_{2}\right)$ if and only if $u_{1}$ is adjacent to $v_{1}$ in $\Gamma_{1}$, or $u_{1}=v_{1}$ and $u_{2}$ is adjacent to $v_{2}$ in $\Gamma_{2}$. The tensor product $\Gamma_{1} \otimes \Gamma_{2}$ is the graph with vertex set $V\left(\Gamma_{1}\right) \times V\left(\Gamma_{2}\right)$, and $\left(u_{1}, u_{2}\right)$ is adjacent to $\left(v_{1}, v_{2}\right)$ if and only if $u_{1}$ is adjacent to $v_{1}$ in $\Gamma_{1}$ and $u_{2}$ is adjacent to $v_{2}$ in $\Gamma_{2}$. The complement of a graph $\Gamma$ is denoted by $\Gamma^{\mathrm{c}}$, and the complete graph with $n$ vertices by $K_{n}$.

The main result of this paper is the following.

Theorem 1.1 Let $\Gamma$ be a connected dihedrant over the dihedral group $D_{2 n}$ which admits an arc-regular action of a subgroup $D_{2 n} \leq G \leq \operatorname{Aut}(\Gamma)$, and moreover, the 
cyclic group of index 2 in $D_{2 n}$ is core-free in $G$. Then

$$
\Gamma \cong\left(K_{n_{1}} \otimes \cdots \otimes K_{n_{\ell}}\right)\left[K_{m}^{c}\right]
$$

where $2 n=m n_{1} \cdots n_{\ell}$ and the numbers $n_{i}$ are pairwise coprime.

Remark Theorem 1.1 gives only a possible structure of the dihedrants $\Gamma$ belonging to the family $\mathcal{F}$. However, we do not know which of the graphs in (1) really do belong to the family $\mathcal{F}$. To the best of our knowledge, the only known examples are $K_{4}$ and $K_{4}\left[K_{2}^{\mathrm{c}}\right]$ (with the corresponding group $G=A_{4}$ and $G=S_{4}$, respectively), and the complete bipartite graphs $K_{n, n} \cong K_{2}\left[K_{n}^{\mathrm{c}}\right]$ (see Sect. 3 for the proof of the latter fact).

The paper is organized as follows. In Sect. 2 we describe minimal blocks of groups $G$ described in (A). In Sect. 3 we prove that the complete bipartite graphs $K_{n, n}$ belong to the family $\mathcal{F}$. Herzog and Kaplan [6] proved that the core ${ }_{G}(C)$ of a cyclic subgroup $C$ in a group $G$ is nontrivial if $[G: C] \leq|C|$. In Sect. 4 we prove a result of this type for dihedral subgroups (Theorem 4.3), which is of independent interest but is also used in the proof of Theorem 1.1 given in Sect. 5. Finally, in Sect. 6 we apply Theorem 1.1 to 1-regular dihedrants and Cayley maps over dihedral groups (in short, dihedral maps). In particular, we give a short proof of the fact that every 1-regular dihedrant of prime valency is normal, a result first proved in [7] (see Theorem 6.3). Moreover, a direct translation of Theorem 1.1 into the language of maps on surfaces gives us that in a chiral dihedral map $\mathcal{M}$ whose automorphism group Aut $(\mathcal{M})$ coincides with the automorphism group of its underlying graph, the index 2 cyclic subgroup in the corresponding regular dihedral subgroup of $\operatorname{Aut}(\mathcal{M})$ has nontrivial core in $\operatorname{Aut}(\mathcal{M})$ (see Corollary 6.4).

\section{Minimal blocks and quotients}

We first set some notation and terminology. Let $G$ be a group acting on a set $X$. For a subset $Y \subseteq X \cup 2^{X} \cup 2^{\left(2^{X}\right)} \cup \cdots, G_{Y}$ denotes the elementwise stabilizer of $Y$ in $G$. Some special cases of this notation: if $Y \subseteq X$, then $G_{Y}$ denotes the elementwise stabilizer of $Y$, while $G_{\{Y\}}$ denotes the setwise stabilizer of $Y$. If $Y, Z \subseteq X$, then $G_{\{Y, Z\}}=\left\{g \in G: Y^{g}=Y, Z^{g}=Z\right\}$. For a $G$-invariant subset $Y \subseteq X$, denote by $g^{Y} \in \operatorname{Sym}(Y)$ the permutation of $Y$ induced by $g \in G$, and set $G^{Y}:=\left\{g^{Y}: g \in G\right\}$. For $Y \subseteq X$, we abbreviate $\left(G_{\{Y\}}\right)^{Y}$ as $G_{\{Y\}}^{Y}$. Suppose that $G$ acts transitively on $X$, and let $\mathcal{B}$ be an imprimitivity system of $G$. Then $G_{\mathcal{B}}:=\left\{g \in G: \forall_{B \in \mathcal{B}} B^{g}=B\right\}$ is the kernel of the action of $G$ induced on $\mathcal{B}$. We say that $\mathcal{B}$ is normal if $G_{\mathcal{B}}$ acts transitively on each block $B \in \mathcal{B}$. A block $B$ of $G$ is a minimal block if it does not contain any nontrivial proper block of $G$, and the imprimitivity system $\mathcal{B}$ of $G$ is minimal if it is generated by a minimal block $B$, i.e., $\mathcal{B}=\left\{B^{g} \mid g \in G\right\}$.

Suppose that, in addition, $G$ contains a regular dihedral subgroup $D,|D|>4$, and let $C \leq D$ denote the corresponding cyclic subgroup. The elements of $D \backslash C$ will be called reflections and those of $C$ rotations. In what follows we write $C_{d}$ for the unique subgroup of $C$ of order $d$. If $B$ is a block of $G$, then $D_{\{B\}}$ is a subgroup of $D$ 
of order $|B|$. We say that $B$ is of cyclic (dihedral resp.) type if $D_{\{B\}} \leq C\left(D_{\{B\}} \not \subset C\right.$ resp.).

Let $\Gamma$ be a $G$-arc-regular graph, and $\mathcal{B}$ be an imprimitivity system of $G$. The quotient $\bar{\Gamma}$ of $\Gamma$ with respect to $\mathcal{B}$ is the graph with vertex set $\mathcal{B}$, and $\left(B, B^{\prime}\right) \in \mathcal{B} \times \mathcal{B}$ is an arc if and only if $\Gamma$ contains an arc $\left(x, x^{\prime}\right)$ with $x \in B$ and $x^{\prime} \in B^{\prime}$. Denote by $\bar{G}$ the permutation group of $\mathcal{B}$ induced by $G$ acting on $\mathcal{B}$. In what follows, to $\bar{G}$ we shall also refer as the quotient of $G$ with respect to $\mathcal{B}$. The particular case that $\Gamma$ is a dihedrant and $\mathcal{B}$ is minimal is analyzed in this section (see Proposition 2.2). For a similar result, we refer to [13, Lemma 6.5].

Let $G \leq \operatorname{Sym}(X)$ be a transitive permutation group, and let $H \leq G$ be a regular subgroup such that $H$ is either cyclic or dihedral. In what follows, we denote by $H^{\star}$ the subgroup generated by the elements of $H$ of the largest order. Thus, if $H$ is dihedral of order greater than 4 , then $H^{\star}$ is the unique cyclic subgroup of order $|H| / 2$, and $H^{\star}=H$ if $H$ is cyclic.

Lemma 2.1 Let $G \leq \operatorname{Sym}(X)$ be a 2-transitive group, and let $H \leq G$ be a regular subgroup, $H$ is cyclic or dihedral, $|H|>4$, and $|H|$ is nonprime. Then each normal subgroup $N \leq G$ which contains $H^{\star}$ is 2-transitive.

Proof If $N$ is elementary abelian of order $p^{d}$, then $|X|=p^{d}$. It follows from $H^{\star} \leq N$ that $\left|H^{*}\right|=p$. Thus, either $|X|=p$ (if $H$ is cyclic) or $|X|=2 p=4$ ( $H$ is dihedral). Both of the cases contradict the assumption that $|H|$ is nonprime and $|H|>4$.

Assume now that $N$ is not elementary abelian. By [25, Exercises 12.3-4], $N$ is primitive. If $H$ is cyclic, then $H=H^{\star} \leq N$, and by Schur's Theorem [25, Theorem 25.3], $N$ is 2-transitive. If $H$ is dihedral and $H \leq N$, then a result of Wielandt [24, Satz 2] yields the result. Consider the remaining case $H \not \leq N$. The group $H N$ is primitive and contains $H$, and we conclude, as before, that $H N$ is 2-transitive. The inclusions $H^{\star}<H$ and $H^{\star} \leq N$ imply that $H \cap N=H^{\star}$, and, therefore, $[H N: N]=2$. Therefore, if $N$ is not 2-transitive, it is of rank three with two nontrivial suborbits of the same length. But in this case the degree of $G$ is odd, contrarily to $|H|$ being even.

Proposition 2.2 Let $\Gamma$ be a connected, G-arc-regular dihedrant over the dihedral group $D_{2 n}$ of order $2 n$, where $D_{2 n} \leq G \leq \operatorname{Aut}(\Gamma)$. Let $C_{d} \leq D_{2 n}$ be a cyclic subgroup, $\left|C_{d}\right|=d$, and let $\mathcal{B}$ be a minimal imprimitivity system having blocks of cardinality $r$. Denote by $\bar{\Gamma}$ and $\bar{G}$ the respective quotient graph and group, respectively. Then either $r$ is a prime or $r=4$, and one of the following holds.

(a) If $r$ is odd, then either $C_{r} \unlhd G$ or $\left\langle C_{r}^{G}\right\rangle \cong \mathbb{Z}_{r}^{2}$, the quotient graph $\bar{\Gamma}$ is $\bar{G}$-arcregular with valency $k / r$, and $\Gamma=\bar{\Gamma}\left[K_{r}^{c}\right]$.

(b) If $r=2$ and $B$ is of cyclic type, then $C_{2} \unlhd G$.

(c) If $r=2$ and $B$ is of dihedral type, then $\bar{\Gamma}$ is a $\bar{G}$-arc-transitive circulant the edge stabilizer of which is a subgroup of $\mathbb{Z}_{2}^{2}$.

(d) If $r=4$, then $B$ is of dihedral type, $\left\langle C_{2}^{G}\right\rangle \cong \mathbb{Z}_{2}^{t}, 2 \leq t \leq 4$, and $\left\langle C_{2}^{G}\right\rangle$ acts transitively on $B$. 
Proof Assume, toward a contradiction, that $r$ is not prime and $r>4$. Let $H \leq D$ be a setwise stabilizer of $B$ in $D$ and $C:=H^{\star}$. Then $C$ is a nontrivial subgroup of $G_{\mathcal{B}}$, and, therefore, $1 \neq C^{B} \leq G_{\mathcal{B}}^{B}$. Thus, $G_{\mathcal{B}}^{B}$ is a nontrivial normal subgroup of $G_{\{B\}}^{B}$ which contains $C^{B}$. Since $B$ is minimal, $G_{\{B\}}^{B}$ is primitive, which, together with $G_{\{B\}}^{B} \geq H_{\{B\}}^{B}$, implies that $G_{\{B\}}^{B}$ is 2-transitive on $B$. Since $G_{\mathcal{B}}^{B} \unlhd G_{\{B\}}^{B}$, Lemma 2.1 implies that $G_{\mathcal{B}}^{B}$ is also 2-transitive on $B$. Let $B^{\prime} \in \mathcal{B}$ be a block connected to $B$ by an edge of $\Gamma$. Then $G_{\mathcal{B}}$ acts semiregularly on the edges of $\Gamma$ between $B$ and $B^{\prime}$. Therefore, $\left|G_{\mathcal{B}}\right| \leq|B|^{2}$. On the other hand, $G_{\mathcal{B}}^{B}$ is a homomorphic image of $G_{\mathcal{B}}$ which acts 2-transitively on $B$. Therefore, $\left|G_{\mathcal{B}}\right| \geq\left|G_{\mathcal{B}}^{B}\right|=m|B|(|B|-1)$. Now the inequality $m|B|(|B|-1) \leq|B|^{2}$ yields $m=1$, that is, $G_{\mathcal{B}}^{B}$ is 2-transitive Frobenius group of order $|B|(|B|-1)$. Hence $G_{\mathcal{B}}^{B}$ contains an elementary abelian normal subgroup, $N$ say, of order $p^{k}=|B|=|H|$. It follows from $\left|G_{\mathcal{B}}^{B}\right|=p^{k}\left(p^{k}-1\right)$ that $N$ is a unique Sylow $p$-subgroup of $G_{\mathcal{B}}^{B}$. Since $C^{B} \leq G_{\mathcal{B}}^{B}$ and the order of $C^{B}$ is either $p^{k}$ or $p^{k} / 2$ (in this case $p=2$ ), we obtain $C^{B} \leq N$. However, $C^{B} \cong C$ is cyclic, while $N$ is elementary abelian, a contradiction.

To prove the rest of the statement, we pick an arbitrary $B^{\prime} \in \mathcal{B}$ connected with $B$ by an edge $\left(x, x^{\prime}\right)$ of $\Gamma\left(x \in B, x^{\prime} \in B^{\prime}\right)$.

CASE (A) $r$ is odd.

In this case $C_{r} \leq G_{\mathcal{B}}$, and the blocks of $\mathcal{B}$ are orbits of $G_{r}$. If $C_{r}^{B}$ is not normal in $G_{\mathcal{B}}^{B}$, then by Burnside's theorem $G_{\mathcal{B}}^{B}$ is 2-transitive. Arguing as before, we obtain that $G_{\mathcal{B}}^{B}$ is a Frobenius group, a contradiction. Thus, $C_{r}^{B} \unlhd G_{\mathcal{B}}^{B}$. Hence a Sylow $r$ subgroup $R$ of $G_{\mathcal{B}}$ is normal in $G_{\mathcal{B}}$ and, therefore, normal in $G$. Clearly $C_{r} \leq R$. It follows from $R_{x y}=1$ that either $|R|=r$ or $|R|=r^{2}$. In the first case, $R=C_{r}$ and we are done. In the second case, $R_{x}$ acts transitively on $B^{\prime}$ implying that $\Gamma \cong \bar{\Gamma}\left[K_{r}^{\mathrm{c}}\right]$. In the latter case factoring over $\mathcal{B}$ we obtain a $\bar{G}$-arc-regular graph $\bar{\Gamma}$ on $2 n / r$ points with valency $k / r$ and $|\bar{G}|=|G| / r^{2}$.

CASE (B) $r=2$, and $B$ is of cyclic type.

In this case $C_{2}$ is the only semiregular subgroup of $G_{\mathcal{B}}$. Therefore, $C_{2} \unlhd G$.

CASE (C) $r=2$ and $B$ is of dihedral type.

In this case $\bar{\Gamma}$ is a circulant. The edge stabilizer of $\bar{\Gamma}$ is a factor-group of $G_{\left\{B, B^{\prime}\right\}}$ over $G_{\mathcal{B}}$. Now the claim follows, since $G_{\left\{B, B^{\prime}\right\}}$ is embedded into $\mathbb{Z}_{2} \times \mathbb{Z}_{2}$.

CASE (D) $r=4$.

Assume, toward a contradiction, that $B$ is of cyclic type. Then $C_{4}^{B}$ is a regular cyclic subgroup contained in $G_{\{B\}}^{B}$. Since $G_{\{B\}}^{B}$ is 2-transitive, we obtain $G_{\{B\}}^{B} \cong S_{4}$. It follows from $C_{4} \leq G_{\mathcal{B}}$ that $G_{\mathcal{B}}^{B}$ is a normal subgroup of $S_{4}$ containing a 4-cycle. Therefore, $G_{\mathcal{B}}^{B} \cong S_{4}$ implying $G_{\mathcal{B}}^{B}=G_{\{B\}}^{B} \cong S_{4}$. Thus, $G_{\mathcal{B}} \cong G_{\mathcal{B}}^{B \cup B^{\prime}}$ is a subdirect product of $S_{4} \times S_{4}$ the order of which is at least 24 . On the other hand, $\left|G_{\mathcal{B}}\right|=$ $\left|\left(x, x^{\prime}\right)^{G_{\mathcal{B}}}\right| \leq 16$, a contradiction. Thus, $B$ is of dihedral type. Since $B$ is minimal, $G_{\{B\}}^{B}$ is primitive, and, therefore $G_{\{B\}}^{B}$ is either $A_{4}$ or $S_{4}$.

The group $C_{2}^{B}$ is nontrivial and is contained in the Klein subgroup of $\operatorname{Sym}(B)$. Therefore, $\left\langle C_{2}^{G}\right\rangle^{B}$ is isomorphic to $\mathbb{Z}_{2}^{2}$ implying that $\left\langle C_{2}^{G}\right\rangle$ is an elementary abelian 
2-group. Now 1-regularity of $G$ implies that $\left\langle C_{2}^{G}\right\rangle \cong \mathbb{Z}_{2}^{t}$ with $t \leq 4$. The inequality $2 \leq t$ follows from $\left\langle C_{2}^{G}\right\rangle^{B} \cong \mathbb{Z}_{2}^{2}$.

\section{An arc-regular action on $K_{n, n}$}

In this section we are going to show that the complete bipartite graphs $K_{n, n}$ belong to family (A). Our argument utilizes the well-known correspondence between $G$-arctransitive graphs and double cosets of a point stabilizer of $G$, a special case of coset graphs, see [1, p. 128].

Let $G$ be any group, and $H \leq G$ be a core-free subgroup. Then the action of $G$ on the set $G / H$ of right $H$-cosets is a faithful permutation representation of $G$. The $G$-arc-transitive digraph $\operatorname{Cos}(G, H, H g H)$, corresponding to the double coset $H g H$, is the digraph with vertex set $G / H$, and arcs are all pairs $(H x, H y)$ such that $x y^{-1} \in H g H$. Note that $\operatorname{Cos}(G, H, H g H)$ is undirected and connected if and only if $H g H=H^{-1} H$ and $\langle H, g\rangle=G$, respectively. Also, it is not difficult to see that $\operatorname{Cos}(G, H, H g H)$ is isomorphic to $K_{n, n}$ (where $2 n=[G: H]$ ) if and only if $H H^{g}$ is a subgroup of $G$ of index 2 .

Proposition 3.1 Let $G$ be a finite group which contains an index two subgroup $N$ such that $N \cong D_{n} \times D_{n}$ where $n=2 m, m$ is odd. If $G / N$ interchanges the direct factors of $N$, then $G \cong\left(D_{n} \times D_{n}\right) \rtimes\langle\sigma\rangle$, where $\sigma$ is an automorphism of $D_{n} \times D_{n}$ interchanging the coordinates (that is, $(x, y)^{\sigma}=(y, x)$ ).

Proof Let $U$ be one of the dihedral subgroups mentioned in the assumption. First we show that $G \backslash N$ contains an element of order 2 . We can always choose $g \in G \backslash N$ to be a 2-element. Since $g^{2} \in N$ and a Sylow 2-subgroup of $N$ is isomorphic to $\mathbb{Z}_{2} \times \mathbb{Z}_{2}$, either $o(g)=2$ or $o(g)=4$. There is nothing to prove in the first case. So we may assume that $o(g)=4$. Then $U^{g^{2}}=U$. Since $U$ has an odd number of involutions, there exists one, say $u$, centralized by $g^{2}$. Since $U^{g}$ and $U$ are trivially intersecting normal subgroups of $N$, the elements $u$ and $u^{g}$ are distinct and commute. Thus, $\left\langle u, u^{g}\right\rangle \cong \mathbb{Z}_{2} \times \mathbb{Z}_{2}$. Since $g$ normalizes $\left\langle u, u^{g}\right\rangle$, the group $\left\langle u, u^{g}\right\rangle\langle g\rangle$ is a 2 group of order at least 8 . But a Sylow 2-subgroup of $G$ has order 8 . Therefore,

$$
\left|\left\langle u, u^{g}\right\rangle\langle g\rangle\right|=8 \quad \Longrightarrow \quad g^{2} \in\left\langle u, u^{g}\right\rangle \quad \Longrightarrow \quad u u^{g} g^{-2}=1 \quad \Longrightarrow \quad\left(u g^{-1}\right)^{2}=1 .
$$

Thus, $u g^{-1}$ is a required element from $G \backslash N$ of order 2 .

Let $s$ be an involution contained in $G \backslash N$. Then $G \cong N \rtimes\langle s\rangle$. Since $G / N$ interchanges the direct factors of $N, N$ is a direct product of $U$ and $U^{s}$. Identifying now $N$ with $U \times U$ via the isomorphism $(u, v) \mapsto u v^{s}$, we obtain that $G \cong\left(D_{n} \times D_{n}\right) \rtimes\langle\sigma\rangle$.

In what follows we write $\mathbb{D}_{n}$ for the group $\left(D_{n} \times D_{n}\right) \rtimes\langle\sigma\rangle$. The elements of $\mathbb{D}_{n}$ will be written as triples $\left(a, b, \sigma^{i}\right)$ where $a, b \in D_{n}$ and $i \in \mathbb{Z}_{2}$. The product of two triples is defined via the action of $\sigma$ on $D_{n} \times D_{n}$ :

$$
\left(a, b, \sigma^{i}\right)\left(c, d, \sigma^{j}\right)= \begin{cases}\left(a c, b d, \sigma^{i+j}\right) & \text { if } i=0 \\ \left(a d, b c, \sigma^{i+j}\right) & \text { if } i=1 .\end{cases}
$$


If $X \leq D_{2 n}$, write $(X, X, 1):=\{(a, b, 1) \mid a, b \in X\}$ and set $N:=\left(D_{2 n}, D_{2 n}, 1\right)$. We fix two involutions $s, t \in D_{n}$ which generate $D_{n}$ and denote $C_{m}:=\langle c\rangle, c:=s t$. The subset $D:=\left\{(a, b, 1) \mid a \in D_{n}, b \in\langle s\rangle\right\}$ is a subgroup of $N$ isomorphic to $D_{n} \times \mathbb{Z}_{2} \cong D_{2 n}$. In what follows we denote by $C$ a unique cyclic subgroup of $D$ of order $n$, that is, $C=\left\{(a, b, 1) \mid a \in C_{m}, b \in\langle s\rangle\right\}$. Let $p$ be a prime divisor of $n=2 m$, and $C_{p}$ be a unique subgroup of $C$ of order $p$. If $p$ is odd, then $C_{p}=\left\langle\left(c^{m / p}, 1,1\right)\right\rangle$ and $\mathrm{N}_{\mathbb{D}_{n}}\left(C_{p}\right)=N$. If $p=2$, then $C_{p}=\langle(1, s, 1)\rangle$. In this case $\mathrm{N}_{\mathbb{D}_{n}}\left(C_{p}\right)=\mathrm{C}_{\mathbb{D}_{n}}((1, s, 1))=\left(D_{n},\langle s\rangle, 1\right)=D \leq N$. Therefore,

$$
\bigcup_{p \in \pi(n)} \mathrm{N}_{\mathbb{D}_{n}}\left(C_{p}\right)=N,
$$

implying that core $\mathbb{D}_{n}(C)$ is trivial.

Proposition 3.2 Every dihedral subgroup of $N$ of order $2 n$ is $\mathbb{D}_{n}$-conjugate to $D$.

Proof Let $F \cong D_{2 n}$ be a subgroup of $N$. Then $F$ contains a unique central involution, say $g=(a, b, 1)$. If $a, b \neq 1$, then $\mathrm{C}_{N}(g)$ has order 4 contrary to $F \leq \mathrm{C}_{N}(g)$. Thus, $a=1$ or $b=1$. In both cases, $g$ is $\mathbb{D}_{n}$-conjugate to $(1, s, 1)$. So, we may assume that $g=(1, s, 1)$. Since $F$ centralizes $g$, we obtain $F \leq \mathrm{C}_{N}(g)=D$. Hence $F=D$.

The connected coset digraphs arising from $\mathbb{D}_{n}$ are determined in the next proposition.

Proposition 3.3 Let $A \leq \mathbb{D}_{n}$ be a core-free subgroup satisfying $A D=\mathbb{D}_{n}$ and $A \cap$ $D=1$. Consider the transitive action of $\mathbb{D}_{n}$ on the set $\mathbb{D}_{n} / A$ of right $A$-cosets. Then every connected arc-transitive digraph of $\mathbb{D}_{n}$ is isomorphic to $K_{n, n}$.

Proof First we classify all the subgroups $A$ satisfying the assumptions of our statement. It follows from $A D=\mathbb{D}_{n}$ and $A \cap D=1$ that $|A|=n=2 \mathrm{~m}$. Since $[G: N]=2$, either $A \leq N$ or $[A: A \cap N]=2$. The first case is impossible because $D \leq N$ and $A D=G$. Therefore $[A: A \cap N]=2$, implying $|A \cap N|=m$. Since $m$ is odd, all involutions of $A$ are contained in $A \backslash(A \cap N) \subset \mathbb{D}_{n} \backslash N$. Let $r=(x, y, \sigma) \in A \backslash(A \cap N)$ be an involution. Then $r^{2}=1$ yields $y=x^{-1}$ and $r=(x, 1,1)(1,1, \sigma)\left(x^{-1}, 1,1\right)$. Therefore $r$ is conjugate in $\mathbb{D}_{n}$ with $(1,1, \sigma)$. So, without loss of generality, we may assume that $r=(1,1, \sigma) \in A$.

Let $\pi$ denote the projection of $N$ onto the second coordinate. Since $\operatorname{ker}(\pi) \leq D$ and $A \cap N \cap D=1$, we obtain $\pi(A \cap N) \cong A \cap N$. In particular, $|\pi(A \cap N)|=m$. Since $\pi(N)$ is a dihedral group of order $2 m, A \cap N \cong \pi(A \cap N)$ is a cyclic group of order $m$. Therefore $A \cap N=\left\langle\left(c^{i}, c^{j}, 1\right)\right\rangle$ for some $i, j \in \mathbb{Z}_{m}$. It follows from $\left|\left\langle\left(c^{i}, c^{j}, 1\right)\right\rangle\right|=m$ that $\operatorname{gcd}(i, j, m)=1$. Since $\left(c^{i}, c^{j}, 1\right)^{o\left(c^{j}\right)} \in D$, we obtain that $o\left(c^{j}\right)=m$ or, equivalently, $\operatorname{gcd}(j, m)=1$. Raising $\left(c^{i}, c^{j}, 1\right)$ into $j^{-1}$ power, we obtain $A \cap N=\left\langle\left(c^{x}, c, 1\right)\right\rangle$ for some $x \in \mathbb{Z}_{m}$. Since $r$ normalizes $A \cap N$, we obtain $\left(c, c^{x}, 1\right) \in\left\langle\left(c^{x}, c, 1\right)\right\rangle$, implying $x^{2} \equiv 1(\bmod m)$. Thus, $A=$ $(A \cap N)\langle r\rangle=\left\{\left(c^{x j}, c^{j}, \sigma^{k}\right) \mid j \in \mathbb{Z}_{m}, k \in \mathbb{Z}_{2}\right\}$. A direct calculation shows that $A A^{(1, s, 1)}=O\langle(s, s, 1),(1,1, \sigma)\rangle$ is a subgroup of $\mathbb{D}_{n}$ of index 2 (where $O:=$ 
$\left.\left(C_{m}, C_{m}, 1\right)\right)$. Thus, the digraph corresponding to the double coset $A(1, s, 1) A$ is isomorphic to $K_{n, n}$.

\section{A Herzog-Kaplan-type theorem}

Herzog and Kaplan [6] proved that the $\operatorname{core}_{G}(C)$ of a cyclic subgroup $C$ in a group $G$ is nontrivial if $[G: C] \leq|C|[6$, Theorem B]. In this section we prove a similar result about dihedral subgroups (Theorem 4.3). We start with the following.

Proposition 4.1 Let $C=\langle c\rangle$ be a cyclic subgroup of $G$. Let $p$ be a prime divisor of $|C|$, and $P \leq C$ be the subgroup of order $p$. Assume that $N:=\left\langle P^{G}\right\rangle$ is an elementary abelian p-group. Then $\left|\operatorname{core}_{G / N}(C N / N)\right| \leq\left|\operatorname{core}_{G}(C)\right|$.

Proof Let $Q \leq C$ be such that core $_{G / N}(C N / N)=Q N / N$. Then $Q \cap N=P$, and, consequently, $P \leq \mathbf{Z}(Q N)$. It follows from $Q N \unlhd G$ that $\mathbf{Z}(Q N) \unlhd G$. Together with $P \leq \mathbf{Z}(Q N)$ and $N=\left\langle P^{G}\right\rangle$, we obtain that $N \leq \mathbf{Z}(Q N)$. But $Q N / N$ is cyclic. Hence, $Q N / \mathbf{Z}(Q N)$ is cyclic too. Therefore, $Q N$ is an abelian normal subgroup of $G$. The subgroup $(Q N)^{p}$ generated by $p$ th powers of $Q N$ is characteristic in $Q N$ and, therefore, normal in $G$. But $(Q N)^{p}=Q^{p}$, implying $Q^{p} \unlhd G \Longrightarrow Q^{p} \leq$ core $_{G}(C)$. Since $Q$ is cyclic, $\left[Q: Q^{p}\right]=p$. It follows from the definition of $Q$ that $|Q|=\left|\operatorname{core}_{G / N}(C N / N)\right| p$. Now the claim follows.

Lemma 4.2 Let $D \leq G$ be a dihedral subgroup of order $2 n, n \geq 3$, and $C \leq D$ its cyclic subgroup of order $n$. If $[G: D] \leq n$ and core $_{G}(C)=1$, then either $[G: D]=n$ and $G \cong \mathbb{D}_{n}$, or $C^{g} \cap C \neq 1$ for each $g \in G$.

Proof Assume that $C^{g_{0}} \cap C=1$ for some $g_{0} \in G$. Consider the intersection $D^{g_{0}} \cap D$. It follows from $C^{g_{0}} \cap C=1$ that $\left|D^{g_{0}} \cap D\right| \leq 4$. If $\left|D^{g_{0}} \cap D\right|=3$, then $\left|C^{g_{0}} \cap C\right|=3$, which is impossible. Therefore, $\left|D^{g_{0}} \cap D\right|=1,2$, 4. If $\left|D^{g_{0}} \cap D\right| \leq 2$, then

$$
|G| \geq|D|+\left|D g_{0} D\right| \geq 2 n+(2 n)^{2} / 2=2 n(n+1),
$$

contrary to the assumption $[G: D] \leq n$. Thus, $\left|D^{g_{0}} \cap D\right|=4$, and, consequently, $\left|D^{g_{0}} \cap C\right|=\left|D \cap C^{g_{0}}\right|=2$. In particular, $n$ is even, and $D^{g_{0}} \cap C=\left\{1, c^{n / 2}\right\}$ where $c$ is a generator of $C$. It follows from $C^{g_{0}} \cap C=1$ that $c^{n / 2}=s^{g_{0}}$ for some $s \in D \backslash C$. Therefore, $\chi\left(c^{n / 2}\right)=\chi(s)$ for any character $\chi$ of $G$.

Let now $\chi$ be the character of the permutation representation of $G$ on the set $G / D$ of right $D$-cosets. Let $O_{0}=\{D\}, O_{1}=\left\{D g_{0} d \mid d \in D\right\}, O_{2}, \ldots, O_{r}$ be the orbits of $D$. Then the stabilizer of $D g_{0}$ in $D$ is $D \cap D^{g_{0}}$, implying that $\left|O_{1}\right|=n / 2$. It follows from

$$
D g_{0} d c^{n / 2}=D g_{0} c^{n / 2} d=D g_{0} s^{g_{0}} d=D g_{0} d, \quad d \in D,
$$

that $c^{n / 2}$ acts trivially on $O_{1}$, implying $\chi\left(c^{n / 2}\right) \geq 1+n / 2$. Since the action of $D$ on $O_{1}$ is equivalent to the natural action of the dihedral group of order $n$ on $n / 2$ points, an element $s$ may fix at most two points of $O_{1}$. If $s$ acts nontrivially on $O_{2} \cup \cdots \cup O_{r}$, 
then it should move at least two points. So, in this case, it could fix at most $\mid \mathrm{O}_{2} \cup$ $\cdots \cup O_{r} \mid-2$ points. But $\left|O_{2} \cup \cdots \cup O_{r}\right| \leq n / 2-1$. Hence, if $s$ acts nontrivially outside $O_{0} \cup O_{1}$, then $\chi(s) \leq n / 2$, contrary to $\chi(s)=\chi\left(c^{n / 2}\right) \geq n / 2+1$. Thus, $s$ acts trivially on $O_{2} \cup \cdots \cup O_{r}$, and $\left|O_{2} \cup \cdots \cup O_{r}\right|=n / 2-2, n / 2-1$.

Let us first show that $n / 2$ is odd. Pick $t \in D \backslash C$ to be a reflection with $s t=c$ (such a reflection exists for any $s \in D \backslash C$ ). Then $c=s t$ restricted on $O_{2} \cup \cdots \cup O_{r}$ acts as $t$, and, therefore, $c^{2}$ acts trivially on the set $O_{2} \cup \cdots \cup O_{r}$. So if $n / 2$ is even, then $c^{n / 2}=\left(c^{2}\right)^{n / 4}$ acts trivially on $O_{2} \cup \cdots \cup O_{r}$, and, therefore, on the whole set $G / D$, implying $c^{n / 2} \in$ core $_{G}(C)$, a contradiction.

If $\left|O_{2} \cup \cdots \cup O_{r}\right|=n / 2-2$, then the inequality $\chi(s) \geq n / 2+1$, together with $O_{2} \cup \cdots \cup O_{r} \subseteq \operatorname{Fix}(s)$, implies that $s$ fixes at least two points of $O_{1}$. Recall that a reflection of a dihedral group given in its natural action, may fix at most two points, which happens only when the number of points is even. Hence $n / 2$ is even, a contradiction.

Thus, $\left|O_{2} \cup \cdots \cup O_{r}\right|=n / 2-1$ with $n / 2$ being odd. In particular, $|G / D|=n$. In this case $s$ has one fixed point on $O_{1}$, which yields $\chi(s)=n / 2+1$. Therefore, $\chi\left(c^{n / 2}\right)=n / 2+1$, implying that $c^{n / 2}$ moves every point of $O_{2} \cup \cdots \cup O_{r}$. As before, we chose $t \in D \backslash C$ such that $s t=c$. Then $D=\langle s, t\rangle$, and the orbits of $D$ on $O_{2} \cup$ $\cdots \cup O_{r}$ coincide with the orbits of $t$. If $t$ has a fixed point on $O_{2} \cup \cdots \cup O_{r}$, then $c^{n / 2}$ also has a fixed point on $O_{2} \cup \cdots \cup O_{r}$, contradicting $\chi\left(c^{n / 2}\right)=n / 2+1$. Thus, $t$ has no fixed points outside $O_{0} \cup O_{1}$. Hence, the orbits of $D$ outside of $O_{0} \cup O_{1}$ have length 2 . This implies that $G$ is a transitive permutation group of degree $n$ (on $G / D$ ) with subdegrees $1, n / 2,2, \ldots, 2$.

We claim that $B_{0}:=O_{0} \cup O_{2} \cup O_{3} \cup \cdots \cup O_{r}$ is a block of $G$. Consider a subgroup $\left\langle c^{2}\right\rangle$. Since each $O_{i}$ is an orbit of $\langle c\rangle$ and $o(c)=n$ with $n / 2$ being odd, we conclude that Fix $\left(\left\langle c^{2}\right\rangle\right)=B_{0}$. If $B_{0} \cap B_{0}^{g} \neq \emptyset$, then both $\left\langle c^{2}\right\rangle$ and $\left\langle c^{2}\right\rangle^{g}$ are subgroups of $G_{D g} \cong$ $D_{2 n}$ for some $D g \in B_{0} \cap B_{0}^{g}$. Since $D_{2 n}$ contains a unique cyclic subgroup of order $n / 2$, we obtain $\left\langle c^{2}\right\rangle=\left\langle c^{2}\right\rangle^{g}$, and, consequently, $B_{0}=B_{0}^{g}$. Thus, $B_{0}$ is a block of $G$ of size $n$. Since $|G / D|=2 n$, the complement of $B_{0}$ is also a block of $G$.

Let $N \leq G$ be a setwise stabilizer of the block $B_{1}$. Then it stabilizes $B_{0}$, and $[G: N]=2$. Since $N$ contains a stabilizer of a point $D$, we obtain $D \leq N$. Denote by $N_{0}$ (resp. $N_{1}$ ) the pointwise stabilizer of $B_{0}$ (resp. $\left.B_{1}\right)$. Then $N_{0}, N_{1}$ are trivially intersecting normal subgroups of $N$. Hence, $\left|N_{0}\right|\left|N_{1}\right| \leq|N|$. Every element of $G \backslash N$ interchanges $N_{0}$ and $N_{1}$. Therefore, $N_{0} \cong N_{1}$, implying $\left|N_{0}\right|^{2} \leq|N| \Longrightarrow\left|N_{0}\right| \leq n$.

A reflection $s$ acts trivially on $B_{0}$. Therefore, $s \in N_{0}$, implying that $\left\langle s^{N}\right\rangle \leq N_{0}$. But $\left\langle s^{N}\right\rangle \geq\left\langle s^{D}\right\rangle$. The subgroup $\left\langle s^{D}\right\rangle$ has order $n$. Therefore, $N_{0}=\left\langle s^{D}\right\rangle \cong D_{n}$, and, consequently, $N \cong N_{0} \times N_{1}$ ( since $|N|=n^{2}$ ). By Proposition $3.1, G \cong \mathbb{D}_{n}$.

The main result of this section is the following theorem.

Theorem 4.3 Let $D \leq G$ be a dihedral subgroup of order $2 n, n>2$, and let $C$ be its cyclic subgroup of order $n$. If $[G: D] \leq n$ and $\operatorname{core}_{G}(C)=1$, then $[G: D]=n$ and $G \cong \mathbb{D}_{n}$.

Proof We proceed by induction on $n$. By Lemma 4.2, either $[G: D]=n$ and $G \cong \mathbb{D}_{n}$, or $C \cap C^{g} \neq 1$ for each $g \in G$. In the first case we are done. Hence, we may assume 
that $C \cap C^{g} \neq 1$ for each $g \in G$. Let $\pi$ be the set of primes dividing $|C|$, and $C_{p} \leq C$ the unique subgroup of order $p, p \in \pi$. Then $G=\bigcup_{p \in \pi} \mathrm{N}_{G}\left(C_{p}\right)$. Let $\pi_{0} \subseteq \pi$ be a minimal subset with the property $G=\bigcup_{p \in \pi_{0}} \mathrm{~N}_{G}\left(C_{p}\right)$. Since core ${ }_{G}(C)$ is trivial, we can write $\left|\pi_{0}\right| \geq 2$. Since a group cannot be a union of two proper subgroups, we obtain that $\left|\pi_{0}\right| \geq 3$. In particular, $n$ is divisible by at least three distinct primes.

Let $q=\max \left\{p \mid p \in \pi_{0}\right\}$, hence, $q \geq 5$. We copy an argument of Herzog and Kaplan [6] to show that the normal closure $N:=\left\langle C_{q}^{G}\right\rangle$ is an elementary abelian group. It is easily verified that $\left|\pi_{0}\right| \leq q-2$. Thus, from [6, Lemma 1] we get that each $q$-element in $G$ is contained in $\bigcap_{p \in \pi_{0}} \mathrm{~N}_{G}\left(C_{p}\right)$. Therefore, each $q$-element in $G$ is contained in $\bigcap_{p \in \pi_{0}} \mathrm{C}_{G}\left(C_{p}\right)$. Let $G_{q}$ be a Sylow $q$-subgroup of $G$, and let $\left\langle G_{q}^{G}\right\rangle$ be its normal closure in $G$. Then $\left\langle G_{q}^{G}\right\rangle \leq \cap_{p \in \pi_{0}} \mathrm{C}_{G}\left(C_{p}\right)$, implying $\left\langle G_{q}^{G}\right\rangle \leq \mathrm{C}_{G}\left(C_{q}\right)$; in particular, $N=\left\langle C_{q}^{G}\right\rangle$ is an elementary abelian group.

Thus, $N \cap C=C_{q}$, and since $C_{q}$ is not normal in $G,|N|=q^{\ell}, \ell \geq 2$. Since $q$ is odd, $D \cap N=C \cap N=C_{q}$. Consider the quotient group $\bar{G}:=G / N$. We have that $\bar{D} \cong D_{2 n / q}$ and $[\bar{G}: \bar{D}]=[G: D] / q^{\ell-1} \leq n / q=|\bar{C}|$. It follows from Proposition 4.1 that core $\bar{G}(\bar{C})=1$. Since $n$ is divisible by at least three primes, $n / q>2$, and we can apply the induction hypothesis. Thus, $[\bar{G}: \bar{D}]=n / q$ and $\bar{G} \cong \mathbb{D}_{n / q}$. It follows from $G=\bigcup_{p \in \pi_{0}} \mathrm{~N}_{G}\left(C_{p}\right)$ that

$$
\bar{G}=\bigcup_{p \in \pi_{0}} \overline{\mathrm{N}_{G}\left(C_{p}\right)}=\left(\bigcup_{p \in \pi_{0} \backslash\{q\}} \overline{\mathrm{N}_{G}\left(C_{p}\right)}\right) \cup \overline{\mathrm{N}_{G}\left(C_{q}\right)} .
$$

It follows from $\overline{\mathrm{N}_{G}\left(C_{p}\right)} \leq \mathrm{N}_{\bar{G}}\left(\overline{C_{p}}\right)$ that

$$
\bar{G}=\left(\bigcup_{p \in \pi_{0} \backslash\{q\}} \mathrm{N}_{\bar{G}}\left(\overline{C_{p}}\right)\right) \cup \overline{\mathrm{N}_{G}\left(C_{q}\right)} .
$$

If $p \neq q$, then $p$ divides $|\bar{C}|$, and $\overline{C_{p}}$ is a subgroup of $\bar{C}$ of order $p$ (note that $\overline{C_{q}}$ is trivial). Therefore, the union $\bigcup_{p \in \pi_{0} \backslash\{q\}} \mathrm{N}_{\bar{G}}\left(\overline{C_{p}}\right)$ is contained in a subgroup of $\bar{G} \cong$ $\mathbb{D}_{n / q}$ of index 2 (see (2)). Since a group cannot be a union of two proper subgroups, we obtain that $\overline{\mathrm{N}_{G}\left(C_{q}\right)}=\bar{G}$. Together with $N \leq \mathrm{N}_{G}\left(C_{q}\right)$, this implies $\mathrm{N}_{G}\left(C_{q}\right)=G$, a contradiction.

As a direct consequence, we obtain the following:

Corollary 4.4 Let $D \leq G$ be a dihedral subgroup of index $m$. Then there exists a normal subgroup $N$ of $G$ contained in $D$ such that $[G: N] \leq 2 m^{2}$.

Proof The statement is immediate if $|D|=4$. So we assume that $|D| \geq 6$. In this case, $D$ contains a unique cyclic subgroup $C$ of index 2 .

Denote $N:=$ core $_{G}(C)$, where $C \leq D$. Then core $_{G / N}(C / N)$ is trivial. If $|C / N|>2$, then by Theorem 4.3 we obtain that

$$
[G / N: C / N]=m \geq|C / N|=[G: N] / 2 m,
$$

implying that $[G: N] \leq 2 m^{2}$. If $|C / N| \leq 2$, then $[G: N]=4 m \leq 2 m^{2}$, as desired. 


\section{Proof of Theorem 1.1}

We turn back to the dihedrants $\Gamma$ in family (A), and we are going to show that these are of the form (1). Observe that Theorem 4.3 implies that $\Gamma$ has valency $d \geq n$, and if $d=n$, then $\Gamma=K_{n, n}$, so the statement is true in this case. Below we handle the case of $d>n$, but three preliminary results are first necessary.

The first result can be deduced from the classification of arc-transitive circulants, see [18, Theorem 3.1] or [8, Theorem 4].

Proposition 5.1 Let $\Gamma$ be a connected, arc-transitive circulant of order $2 n$ and of valency $d>n$. Then

$$
\Gamma \cong\left(K_{n_{1}} \otimes \cdots \otimes K_{n_{\ell}}\right)\left[K_{m}^{\mathrm{c}}\right],
$$

where $2 n=m n_{1} \cdots n_{\ell}$, and the numbers $n_{i}$ are pairwise coprime.

Proposition 5.2 Let $G$ be a group acting transitively on a set $X$, and let $N$ be an arbitrary normal subgroup acting nonsemiregularly on $X$. Then for each $x \in X$, the set $\operatorname{Fix}\left(N_{x}\right)$ is a proper block of $G$.

Proof Note that $\left|N_{x}\right|$ does not depend on the choice of $x$. If $z \in \operatorname{Fix}\left(N_{x}\right) \cap \operatorname{Fix}\left(N_{y}\right)$, then $N_{x} \leq N_{z}$ and $N_{y} \leq N_{z}$, implying $N_{x}=N_{z}=N_{y}$. Thus, $\operatorname{Fix}\left(N_{x}\right)=\operatorname{Fix}\left(N_{y}\right)$. The set $N_{x}$ is a proper subset because $N$ acts nonsemiregularly on $X$.

We remark that it can happen that $\operatorname{Fix}\left(N_{x}\right)=\{x\}$.

Let $G \leq \operatorname{Sym}(X)$ contain a regular, normal, elementary abelian subgroup of or$\operatorname{der} p^{d}$. Now if $G$ contains a full cycle, i.e., a permutation whose cyclic decomposition is one cycle of length $p^{d}$, then $d=1$ or $d=p=2$, see [17]. This implies the following result.

Proposition 5.3 Let $G \leq \operatorname{Sym}(X)$ be a transitive permutation group containing a full cycle. If $N \leq G$ is an elementary abelian normal p-subgroup, then the orbits of $N$ are of length $p$ or 4 .

Everything is prepared to prove Theorem 1.1.

Proof (Theorem 1.1) It is carried out by induction on the order of the graph. By the above discussion, we may assume that $\Gamma$ is of valency $d>n$. The statement is evident if $G$ is primitive. So, we may assume that $G$ is imprimitive. Let $B$ be a minimal nontrivial block of $G, r:=|B|, \mathcal{B}:=\left\{B^{g} \mid g \in G\right\}$ the corresponding imprimitivity system, $N:=G_{\mathcal{B}}, \bar{G}$ and $\bar{\Gamma}$ the quotient group and graph, respectively (see Sect. 2), and let $B^{\prime} \in \mathcal{B}$ be a block connected with $B$ by an arc of $\Gamma$, say $\left(b, b^{\prime}\right)$. By Proposition 2.2, either $r$ is a prime, or $r=4$.

If $r$ is an odd prime, then part (a) of Proposition 2.2 and the assumption core $_{G}\left(C_{n}\right)=1$ imply that $\bar{\Gamma}$ is a $\bar{G}$-arc-regular connected dihedrant of valency $d / r>n / r$. Since $\left\langle C_{r}^{G}\right\rangle \leq N$ and $C_{r}$ is not normal in $G$, part (a) of Proposition 2.2 implies that $\left\langle C_{r}^{G}\right\rangle$ is an elementary abelian group of order $r^{2}$. Now Proposition 4.1 
yields that core $\bar{G}\left(\overline{C_{n}}\right)$ is trivial. Thus, the induction hypothesis is applicable, and the result follows.

If $r=2$, then $B$ should be of dihedral type, since, otherwise $C_{2} \unlhd G$ (see part (b) of Proposition 2.2), contradicting the assumption core ${ }_{G}\left(C_{n}\right)=1$. The group $G_{\left\{B, B^{\prime}\right\}}$ is a subgroup of $\mathbb{Z}_{2}^{2}$. Therefore, the number of $\operatorname{arcs}$ of $\Gamma$ from the block $B$ to the block $B^{\prime}$ is 1,2 , or 4 . On the other hand, the inequalities

$$
\begin{aligned}
n & <d \quad \text { and } \\
|D(\bar{\Gamma})| & =|D(\Gamma)| /\left|D(\Gamma) \cap\left(B \times B^{\prime}\right)\right|=d \cdot 2 n /\left|D(\Gamma) \cap\left(B \times B^{\prime}\right)\right| \leq n(n-1)
\end{aligned}
$$

imply that $3 \leq\left|D(\Gamma) \cap\left(B \times B^{\prime}\right)\right|$. Thus, $\left|D(\Gamma) \cap\left(B \times B^{\prime}\right)\right|=4$, implying that $\Gamma=\bar{\Gamma}\left[K_{2}^{\mathrm{c}}\right]$. In particular, $\bar{d}=d / 2>n / 2$. The quotient graph $\bar{\Gamma}$ is a circulant. Now the claim follows from Proposition 5.1.

We are left with the case $r=4$ (note that, in this case, $n$ is even). We may assume that $\mathcal{B}$ is a unique minimal imprimitivity system of $G$. Indeed, if $\mathcal{A}$ is another one, then it should be of order 4 (otherwise we can use induction factoring over $\mathcal{A}$ ). By Proposition 2.2, $\mathcal{B}$ and $\mathcal{A}$ are of dihedral type. But an intersection of two imprimitivity systems of order 4 and dihedral type is an imprimitivity system of order 2 , which contradicts the minimality of $\mathcal{B}$. Thus, $\mathcal{B}$ is a unique minimal imprimitivity system of $G$. Hence, any block $A$ of $G$ which intersects $B$ nontrivially must contain $B$.

By part (d) of Proposition 2.2, $B$ is of dihedral type, and $\left\langle C_{2}^{G}\right\rangle$ is an elementary abelian group of order $2^{t}, 2 \leq t \leq 4$. Since $\left\langle C_{2}^{G}\right\rangle$ acts transitively on the blocks of $\mathcal{B}$, the imprimitivity system $\mathcal{B}$ is normal. Therefore, $\left|\Gamma(x) \cap B^{\prime}\right|$ does not depend on the choice of $b \in B$. Let us denote this number by $\ell$. Then $\left|D(\Gamma) \cap\left(B \times B^{\prime}\right)\right|=4 \ell$, and

$$
|D(\bar{\Gamma})|=|D(\Gamma)| /\left|D(\Gamma) \cap\left(B \times B^{\prime}\right)\right|=\frac{d \cdot 2 n}{4 \ell}>\frac{n^{2}}{2 \ell} .
$$

Together with $|D(\bar{\Gamma})| \leq \frac{n}{2}\left(\frac{n}{2}-1\right)$, this implies $\ell>2$. Thus, $\ell=3,4$.

If $\ell=4$, then $\Gamma=\bar{\Gamma}\left[K_{4}^{\mathrm{c}}\right]$. The quotient graph $\bar{\Gamma}$ is a circulant of order $n / 2$ and valency $d / 4>\frac{1}{2}(n / 2)$. By Proposition $5.1, \bar{\Gamma}$ is of the required form.

We complete the proof by showing that the case $\ell=3$ is impossible. In this case the induced subgraph $\Gamma_{B \cup B^{\prime}}$ is $K_{4,4}$ minus a matching. Therefore, $G_{\left\{B, B^{\prime}\right\}}$ acts faithfully on $B$. Now the inclusions $\left\langle C_{2}^{G}\right\rangle \leq N \leq G_{\left\{B, B^{\prime}\right\}}$ imply that both $\left\langle C_{2}^{G}\right\rangle$ and $N$ are faithful on $B$, implying $\left\langle C_{2}^{G}\right\rangle \cong \mathbb{Z}_{2}^{2}$. Since $G_{\left\{B, B^{\prime}\right\}}$ is embedded into $S_{4}$ and $\left|G_{\left\{B, B^{\prime}\right\}}\right|=\left|D(\Gamma) \cap\left(B \times B^{\prime}\right)\right|=12$, we obtain $G_{\left\{B, B^{\prime}\right\}} \cong A_{4}$. Thus, there are two possibilities: either $N=\left\langle C_{2}^{G}\right\rangle \cong \mathbb{Z}_{2}^{2}$, or $N=G_{\left\{B, B^{\prime}\right\}} \cong A_{4}$.

In the second case, $\operatorname{Fix}\left(N_{b}\right)$ is a proper block of $G$ (Proposition 5.2). Since $\left|N_{b}\right|=3$ and $A_{4}$ contains four pairwise conjugate subgroups of order 3 , there are four blocks of that type. Hence, $\left|\operatorname{Fix}\left(N_{b}\right)\right|=n / 2$. Since $N_{b}$ acts on $B$ as a 3-cycle, $B \cap \operatorname{Fix}\left(N_{b}\right)=\{b\}$. But this contradicts the assumption that $\mathcal{B}$ is a unique minimal imprimitivity system of $G$. Hence, $N=\left\langle C_{2}^{G}\right\rangle \cong \mathbb{Z}_{2}^{2}$.

Since $N$ acts semiregularly and the orbits of $N$ form a minimal imprimitivity system (which is $\mathcal{B}$ ), $N$ is a minimal normal subgroup of $G$. Write $N=\left\{1, x_{1}, x_{2}, x_{3}\right\}$, where $x_{1}:=c^{n / 2}$ (note that $x_{1} x_{2} x_{3}=1$ ). The set $\left\{x_{1}, x_{2}, x_{3}\right\}$ is a conjugacy class of $G$. Hence, $K_{i}:=C_{G}\left(x_{i}\right)$ is a subgroup of $G$ of index 3 . If $i \neq j$, then $K_{i} \cap K_{j}=$ 
$C_{G}(N) \unlhd G$. Since the action of $G$ on $\left\{x_{1}, x_{2}, x_{3}\right\}$ is equivalent to either $A_{3}$ or $S_{3}$, the index of $C_{G}(N)$ in $K_{i}$ is either one or two.

To ease the notation, we will write $K$ instead of $K_{1}$. Since $K$ centralizes $x_{1}$, the group $K_{\{B\}}^{B}$ centralizes $x_{1}^{B}$. Since $x_{1}^{B}$ has cyclic type $(2,2)$, its centralizer in $\operatorname{Sym}(B)$ is a 2-group. Thus, $K_{\{B\}}^{B}$ is a 2-group. It follows from $K_{\left\{B, B^{\prime}\right\}} \leq K_{\{B\}}$ that $K_{\left\{B, B^{\prime}\right\}}^{B}$ is a 2-group as well. Since $K_{\left\{B, B^{\prime}\right\}}^{B \cup B^{\prime}}$ is a subdirect product of $K_{\left\{B, B^{\prime}\right\}}^{B}$ and $K_{\left\{B, B^{\prime}\right\}}^{B^{\prime}}$ and both of them are 2-groups, $K_{\left\{B, B^{\prime}\right\}}^{B \cup B^{\prime}}$ is a 2-group. By $G$-arc-regularity, the action of $K_{\left\{B, B^{\prime}\right\}}$ on $B \cup B^{\prime}$ is faithful. Therefore, $K_{\left\{B, B^{\prime}\right\}}$ is a 2-group. Now it follows from $N_{\left\{B, B^{\prime}\right\}} \leq K_{\left\{B, B^{\prime}\right\}} \leq G_{\left\{B, B^{\prime}\right\}}$ and $\left[G_{\left\{B, B^{\prime}\right\}}: N_{\left\{B, B^{\prime}\right\}}\right]=3$ that $N_{\left\{B, B^{\prime}\right\}}=K_{\left\{B, B^{\prime}\right\}}$, implying $N=K_{\left\{B, B^{\prime}\right\}}$.

Consider now the factor-group $\bar{K}:=K / N$ which acts faithfully on $\mathcal{B}$. The cyclic subgroup $\overline{C_{n}} \cong C_{n / 2}$ acts regularly on $\mathcal{B}$. It follows from $N=K_{\left\{B, B^{\prime}\right\}}$ that $\bar{K}_{\left\{B, B^{\prime}\right\}}$ is trivial. We claim that $\bar{\Gamma}$ is a $\bar{K}$-arc-regular graph. Since $\left(B, B^{\prime}\right)$ is an arc of $\bar{\Gamma}$ and its stabilizer $\bar{K}_{\left\{B, B^{\prime}\right\}}$ is trivial, it is sufficient to show that $|\bar{K}|=|D(\bar{\Gamma})|$. Indeed,

$$
|\bar{K}|=|K| / 4=|G| / 12=|D(\Gamma)| / 12=|D(\Gamma)| /\left|D(\Gamma) \cap\left(B \times B^{\prime}\right)\right|=|D(\bar{\Gamma})| .
$$

Thus, $\bar{\Gamma}$ is a $\bar{K}$-arc-regular circulant. Therefore, $\left|\bar{K}_{B}\right|<|\mathcal{B}|-1=n / 2-1=$ $\left|\overline{C_{n}}\right|-1$. By the Kaplan-Herzog result, core $\bar{K}\left(\overline{C_{n}}\right)$ is nontrivial. Let $p$ denote the maximal prime dividing $\mid$ core $_{\bar{K}}\left(\overline{C_{n}}\right) \mid$.

CASE A. $p>2$.

In this case, $C_{2 p} N \unlhd K$. Since $C_{2 p} \cap N=C_{2}$, the group $C_{2 p} N$ is abelian. Therefore, its Sylow $p$-subgroup, $C_{p}$, is normal in $K$. Moreover, $C_{p}$ is contained in $C_{G}(N)$, and, therefore, $C_{p} \unlhd C_{G}(N)$. The orbits of $C_{p}$ form an imprimitivity system, say $\mathcal{A}$ of $C_{G}(N)$. To get a contradiction, it is enough to show that $\mathcal{A}$ is an imprimitivity system of $G$ (recall that $G$ has a unique minimal nontrivial imprimitivity system, $\mathcal{B}$ ). Since $C_{G}(N) \unlhd G$, the conjugate partition $\mathcal{A}^{g}, g \in G$, is also an imprimitivity system of $C_{G}(N)$ of order $p$. We claim that $C_{G}(N)$ cannot have more than one imprimitivity system of order $p$. The subgroup $C_{D_{2 n}}(N)$ centralizes $c^{n / 2} \in N$. So, it can permute (by conjugation) only two elements of $N \backslash C_{2}$. Therefore, $\left[D_{2 n}: C_{D_{2 n}}(N)\right] \leq 2$, and, consequently, $C_{D_{2 n}}(N)$ acts semiregularly with one or two orbits on $V(\Gamma)$. Since $C_{p}$ is a unique normal subgroup of $C_{D_{2 n}}(N)$ of prime order $p>2$, any $C_{D_{2 n}}(N)$-invariant imprimitivity system having blocks of size $p$ should coincide with the orbits of $C_{p}$. So, $\mathcal{A}$ is unique as imprimitivity system of $C_{G}(N)$ of order $p$.

CASE B. $p=2$.

As before, $C_{4} N$ is a normal subgroup of $K$ of order 8 . Write $z_{1}:=c^{n / 4}$. Then $C_{4} N=\left\langle z_{1}\right\rangle N$. Since $\left\langle\overline{z_{1}}\right\rangle \triangleleft \bar{K}$, we obtain $\overline{z_{1}} \in \mathrm{Z}(\bar{K})$. Since $K, K_{2}, K_{3}$ are pairwise conjugate in $G$, there exist $z_{i} \in K_{i}$ such that $x_{i}=z_{i}^{2}$ and $\overline{z_{i}} \in \mathrm{Z}\left(\overline{K_{i}}\right)$. Since $G$ permutes the subgroups $K_{i}$, the elements $\overline{z_{1}}, \overline{z_{2}}, \overline{z_{3}}$ are either equal or pairwise distinct. In the first case, $C_{4} N \unlhd G$. The subgroup $C_{4} N$ is either abelian or dihedral. In both cases the subgroup generated by the square elements $\left(C_{4} N\right)^{(2)}=C_{2} \unlhd G$, a contradiction. Thus, the $\overline{z_{i}}$ 's are pairwise distinct. The set $\left\{\overline{z_{1}}, \overline{z_{2}}, \overline{z_{3}}\right\}$ is a conjugacy 
class of $\bar{G}$. Therefore, $\bar{Z}:=\left\langle\overline{z_{1}}, \overline{z_{2}}, \overline{z_{3}}\right\rangle \unlhd \bar{G}$. It follows from $K \cap K_{2}=K_{2} \cap K_{3}=$ $K_{3} \cap K=C_{G}(N) \geq N$ that $\bar{K} \cap \overline{K_{2}}=\overline{K_{2}} \cap \overline{K_{3}}=\overline{K_{3}} \cap \bar{K}=\overline{C_{G}(N)}$. Therefore, $\bar{Z} \leq C_{\bar{G}}\left(\overline{C_{G}(N)}\right)$.

The orbits of $C_{G}(N)$ form an imprimitivity system for $G$. Since [ $D_{2 n}: C_{D_{2 n}}(N)$ ] $\leq 2$, the number of $C_{G}(N)$-orbits is at most two. If $C_{G}(N)$ has exactly two orbits, then we get a $G$-invariant imprimitivity system of index 2 , which is impossible because $d>n$. Thus, $C_{G}(N)$ is transitive on $V(\Gamma)$, and, therefore, $\overline{C_{G}(N)}$ is transitive on $\mathcal{B}$. This implies that $\bar{Z}:=\left\langle\overline{z_{1}}, \overline{z_{2}}, \overline{z_{3}}\right\rangle$ acts semiregularly on $\mathcal{B}$. So each orbit of $\bar{Z}$ has cardinality $2 m$ where $2 m:=|\bar{Z}|$ and $2 m \mid \frac{n}{2}$.

CASE B1. $n / 4$ is odd.

In this case, also $m$ is odd. Therefore, $\left[\overline{z_{i}}, \overline{z_{j}}\right] \neq 1$ for $i \neq j$ (otherwise $\overline{z_{i}}, \overline{z_{j}}$ generate a subgroup of $\bar{Z}$ of order divisible by 4$)$. Since $\overline{z_{i}} \neq \overline{z_{j}}$ for $i \neq j$, we have $m>1$. Since $\left\{\overline{z_{1}}, \overline{z_{2}}, \overline{z_{3}}\right\}$ is a conjugacy class of $\bar{G}$, we obtain

$$
{\overline{z_{2}}}^{\overline{z_{1}}}=\overline{z_{3}}, \quad \overline{z_{2}} \overline{z_{3}}=\overline{z_{1}}, \quad \overline{z_{2}} \overline{z_{3}}=\overline{z_{1}} .
$$

Therefore, $\overline{z_{1}} \cdot \overline{z_{2}}=\overline{z_{3}} \cdot \overline{z_{1}}=\overline{z_{2}} \cdot \overline{z_{3}}$. Denote $\bar{y}:=\overline{z_{1} z_{2}}$. Then

$$
\left\langle\overline{z_{1}}, \overline{z_{2}}\right\rangle=\left\langle\overline{z_{1}}, \bar{y}\right\rangle=\left\langle\overline{z_{1}}, \overline{z_{3}}\right\rangle \cong D_{2 m},
$$

implying $\bar{Z} \cong D_{2 m}$ and $o(\bar{y})=m$. Since $m$ is odd, the conjugacy class of $\overline{z_{1}}$ in $\bar{Z}$ has size $m$. But $\left\{\overline{z_{1}}, \overline{z_{2}}, \overline{z_{3}}\right\}$ is a conjugacy class of $\bar{G}$. Hence, $m=3$. It follows from $\langle\bar{y}\rangle$ char $\bar{Z} \unlhd \bar{G}$ that $\langle\bar{y}\rangle \unlhd \bar{G}$. Therefore, the orbits of $\langle\bar{y}\rangle$ form an imprimitivity system of $\bar{G}$, say $\mathcal{A}$. A Sylow 3 -subgroup $\bar{P}$ of $\bar{G}_{\mathcal{A}}$ is an elementary abelian normal 3 -subgroup of $\bar{G}_{\mathcal{A}}$. Therefore, $\bar{P} \unlhd \bar{G}$. Since $\bar{c}$ acts regularly on $\mathcal{B}$, the blocks of $\mathcal{A}$ are also orbits of $\left\langle\bar{c}^{n / 6}\right\rangle$. Therefore, $\left\langle\bar{c}^{n / 6}\right\rangle \leq \bar{P}$. If $\left\langle\bar{c}^{n / 6}\right\rangle=\langle\bar{y}\rangle$, then $\left\langle\bar{c}^{n / 6}\right\rangle \unlhd \bar{G}$, and 3 divides $\mid$ core $\bar{K}\left(\overline{C_{n}}\right) \mid$, contrary to the assumption $p=2$. Thus, $\left\langle\bar{c}^{n / 6}\right\rangle \cap\langle\bar{y}\rangle=1$, and, consequently, $|\bar{P}| \geq 9$. Let $P$ denote the preimage of $\bar{P}$ in $G$. Then $P \unlhd G$ and $P=N P_{3}$, where $P_{3}$ is a Sylow 3 -subgroup of $P$. Since $\left|P_{3}\right| \geq 9$ and $|N|=4, \mathrm{O}_{3}(P)$ is nontrivial. Therefore, $G$ contains a normal 3-subgroup. The orbits of this subgroup form an imprimitivity system the order of which is a power of 3 . But this contradicts the assumption that $\mathcal{B}$ is a unique minimal nontrivial imprimitivity system of $G$.

CASE B2. $8 \mid n$.

Since $\bar{c}$ centralizes $\overline{z_{1}}$, the set $\left\{\overline{z_{2}}, \overline{z_{3}}\right\}$ is $\bar{c}$-invariant. Therefore, $\bar{c}^{2}$ centralizes each $\overline{z_{i}}, i=1,2,3$. Now it follows from $\overline{z_{1}}=\bar{c}^{n / 4}=\left(\bar{c}^{2}\right)^{n / 8}$ that $\overline{z_{1}}$ commutes with $\overline{z_{2}}$ and $\overline{z_{3}}$. Therefore, all the elements $\overline{z_{1}}, \overline{z_{2}}, \overline{z_{3}}$ pairwise commute, which yields $\bar{Z} \cong \mathbb{Z}_{2}^{t}$ where $t=2,3$. Since $t=3$ contradicts Proposition 5.3, we obtain that $t=2$.

It follows from $\left[\overline{z_{2}}, \overline{z_{1}}\right]=1,\left[\overline{z_{3}}, \overline{z_{1}}\right]=1$ that $\overline{z_{2}}, \overline{z_{3}} \in \bar{K}$. So, $\bar{Z} \unlhd \bar{K}$. Let $\mathcal{A}$ be the set of orbits of $\bar{Z}$. Then $\mathcal{A}$ is an imprimitivity system of $\bar{K}$, and, therefore, $\mathcal{A}$ also is a complete set of orbits of $\bar{c}^{n / 8}$. Thus, $\left\langle\bar{c}^{n / 8}\right\rangle$ and $\bar{Z}$ are contained in $\bar{M}:=(\bar{K})_{\mathcal{A}}$. Let $A$ be a block of $\mathcal{A}$. Since $\left\langle\bar{c}^{n / 8}\right\rangle^{A}$ is a regular cyclic group of order 4 and $\bar{Z}^{A}$ is a regular elementary abelian group of order 4 , the restriction of $\bar{M}^{A}$ has order at least 8 . On the other hand, $\left[\overline{z_{1}}, \bar{K}\right]=1$ implies $\bar{M}^{A} \leq \mathrm{C}_{\text {Sym(A) }}\left({\overline{z_{1}}}^{A}\right) \cong D_{8}$. Thus, $\bar{M}^{A} \cong D_{8}$.

Let $A_{1} \in \mathcal{A}$ be a block connected to $A$ by an edge of $\bar{\Gamma}$, say $\left(B, B^{\prime}\right)$ (recall that the vertices of $\bar{\Gamma}$ are the blocks of $\mathcal{B})$. Since $\bar{\Gamma}$ is a $\bar{K}$-arc-regular graph, $|\bar{M}| \leq 16$. 
Together with $\bar{M}^{A} \cong D_{8}$, this gives us the two possibilities that either $|\bar{M}|=16$ or $|\bar{M}|=8$. The graph $\bar{\Gamma}$ has valency $d / 3>n / 3>n / 4=|V(\bar{\Gamma})| / 2$. Therefore, $\bar{\Gamma}$ cannot be bipartite. This implies that $\bar{K}$ has no block of index 2 . We will show that in every case, $\bar{K}$ has an imprimitivity system of index 2 , which leads to a contradiction.

Consider first the case of $|\bar{M}|=16$. It follows from $\left|\bar{M}^{A}\right|=8$ that $\bar{M}_{A}$ (the pointwise stabilizer of $A$ in $\bar{M}$ ) has order 2. Since $\bar{M}_{A} \unlhd \bar{M}$ and $\bar{M}$ is a 2-group, $\bar{M}_{A} \leq \mathrm{Z}(\bar{M})$. Together with $\overline{z_{1}} \in \mathrm{Z}(\bar{M})$, we obtain $|\mathrm{Z}(\bar{M})| \geq 4$. Since $\bar{M}$ is nonabelian, the factor-group over the center cannot be cyclic. Therefore, $|\mathrm{Z}(\bar{M})| \leq 4$, implying $|\mathrm{Z}(\bar{M})|=4$, and so that $\mathrm{Z}(\bar{M})=\left\langle\overline{z_{1}}, \bar{M}_{A}\right\rangle$. Since $\mathrm{Z}(\bar{M})$ char $\bar{M}, \mathrm{Z}(\bar{M}) \unlhd \bar{K}$. Since the pointwise stabilizer $\bar{M}_{A}$ is contained in $\mathrm{Z}(\bar{M})$, the group $\mathrm{Z}(\bar{M})$ is not semiregular. Therefore, by Proposition 2.2, $\operatorname{Fix}\left(Z(\bar{M})_{B}\right)$ is a proper block of $\bar{K}$. Since $\mathrm{Z}(\bar{M}) \cong \mathbb{Z}_{2}^{2}$, there are three subgroups of order 2 . The subgroup $\left\langle\overline{z_{1}}\right\rangle$ is semiregular. Therefore, the index of $\operatorname{Fix}\left(Z(\bar{M})_{B}\right)$ is at most 2 . Since the block is proper, its index is at least 2. Finally, $\operatorname{Fix}\left(\mathrm{Z}(\bar{M})_{B}\right)$ has index 2 , as required.

Let now $|\bar{M}|=8$. Then $\bar{M} \cong \bar{M}^{A} \cong D_{8}$. So, $\bar{M}$ contains three subgroups of in$\operatorname{dex} 2$ :

1. the cyclic subgroup $\left\langle\bar{c}^{n / 8}\right\rangle \cong \mathbb{Z}_{4}$ acting semiregularly on $\mathcal{B}$;

2. the subgroup $\bar{Z} \cong \mathbb{Z}_{2}^{2}$ acting semiregularly on $\mathcal{B}$;

3. an elementary abelian subgroup, say $\bar{L}$, of order 4 acting not-semiregularly on $\mathcal{B}$.

It is clear from this description that conjugating by elements of $\bar{K}$ cannot interchange these subgroups. Therefore, each of them is normal in $\bar{K}$. In particular, $\bar{L} \unlhd \bar{K}$. The group $\bar{L}$ contains three involutions. One of them, $\overline{z_{1}}$, is fixed-pointfree. Therefore, $\operatorname{Fix}\left(\bar{L}_{B}\right)$ has index at most 2 . Since $\bar{L}$ is not semiregular, the block $\operatorname{Fix}\left(\bar{L}_{B}\right)$ has index exactly 2 . Thus, $\bar{K}$ has an imprimitivity systems with two blocks, as required, and by this the proof of Theorem 1.1 is completed.

\section{On 1-regular dihedrants and associated chiral dihedral maps}

Given an arbitrary group $G$, recall that a Cayley digraph Cay $(G, S)$ is said to be a normal Cayley digraph of $G$ if the right regular representation, denoted here by $\hat{G}$, is normal in $\operatorname{Aut}(\operatorname{Cay}(G, S))$. Observe that $\hat{G} \leq \operatorname{Aut}(\operatorname{Cay}(G, S))$. For a Cayley digraph Cay $(G, S)$, let Aut $(G, S)=\left\{\alpha \in \operatorname{Aut}(G) \mid S^{\alpha}=S\right\}$ be the group of automorphisms of $G$ which fix $S$ setwise. Then $\operatorname{Aut}(G, S)$ is clearly a subgroup of the stabilizer of the identity element $e \in G$ in $\operatorname{Aut}(\operatorname{Cay}(G, S))$. Xu [27] characterized the normal Cayley digraphs as follows.

Proposition 6.1 A Cayley digraph $\operatorname{Cay}(G, S)$ is normal if and only if $\operatorname{Aut}(G, S)$ is the stabilizer of the identity element $e \in G$ in $\operatorname{Aut}(\operatorname{Cay}(G, S))$.

As an application of Theorem 1.1, we give in Theorem 6.3 below a short proof of a result due to Kim, Kwon, and Lee [7], mentioned as Proposition 4.1 in [11].

The following proposition, interesting on its own, will be used in that respect. 
Proposition 6.2 Let $D \leq S_{2 n}, n \geq 3$, be a regular dihedral group of degree $2 n$, and let $C$ be its cyclic subgroup of order $n$. If $G$ is a group satisfying $D \leq G \leq S_{2 n}$ and $C \triangleleft G$, then $D$ is normal in $G$.

Proof Our claim is equivalent to saying that $\mathbf{N}_{S_{2 n}}(C)=\mathbf{N}_{S_{2 n}}(D)$. The inclusion $\mathbf{N}_{S_{2 n}}(D) \leq \mathbf{N}_{S_{2 n}}(C)$ follows from the fact that $C$ is a characteristic subgroup of $D$. Since $D$ is regular, we have that $\left|\mathbf{N}_{S_{2 n}}(D)\right|=|D| \mid$ Aut $(D) \mid=2 n \cdot n \varphi(n)$, where $\varphi(n)$ is the Euler function. Since $C$ is semiregular with two orbits, we obtain $\left|\mathbf{C}_{S_{2 n}}(C)\right|=2 n^{2}$, and, therefore, $\left|\mathbf{N}_{S_{2 n}}(C)\right|=\left|\mathbf{C}_{S_{2 n}}(C)\right| \mid$ Aut $(C) \mid=2 n^{2} \cdot \varphi(n)$. Now the claim follows.

\section{Theorem 6.3 Every 1-regular dihedrant of prime valency is normal.}

Proof Let $\Gamma=\operatorname{Cay}\left(D_{2 n}, S\right)$ be a 1-regular dihedrant of prime valency $p$, where $D_{2 n}=\left\langle a, b \mid a^{n}=b^{2}=b a b a=1\right\rangle$. We may assume that $p>2$. For $g \in D_{2 n}$, we denote by $\hat{g}$ the permutation of $D_{2 n}$ acting according to the rule $\hat{g}: x \mapsto x g$. Thus, $\hat{D}_{2 n}=\left\{\hat{g} \mid g \in D_{2 n}\right\}$. Let $\hat{C}_{n}=\langle\hat{a}\rangle$. Further, we put $A=\operatorname{Aut}(\Gamma)$ and $N=$ core $_{A}\left(\hat{C}_{n}\right)$.

Let us consider the action of $A$ on the set $A / \hat{D}_{2 n}$ of right $\hat{D}_{2 n}$-cosets in $A$. Let $K$ be the corresponding kernel, core ${ }_{A}\left(\hat{D}_{2 n}\right)$, and, of course, $\hat{D}_{2 n}$ is the point stabilizer of this action. Therefore, $p$ does not divide the index $\left[\hat{C}_{n}: N\right]$. The orbits of $N$ form an imprimitivity system of $A$; denote it by $\mathcal{B}$. Let $\bar{\Gamma}$ be the quotient of $\Gamma$ with respect to $\mathcal{B}$, and let $\bar{A}$ be the permutation group $\bar{A} \leq \operatorname{Sym}(\mathcal{B})$ induced by the action of $A$ on $\mathcal{B}$. Let us assume that $N$ is a proper subgroup of $\hat{C}_{n}$. Then $\bar{\Gamma}$ is of valency $p$. Further, $\bar{\Gamma}$ is connected, $\bar{A}$-arc-regular, and core $\bar{A}\left(\hat{C}_{n}\right)=1$. By Theorem 1.1 , it follows that either $\bar{\Gamma} \cong K_{2}\left[K_{p}^{\mathrm{c}}\right]$ or $\bar{\Gamma} \cong K_{p+1}$. In the first case, $\left[\hat{C}_{n}: N\right]=p$, a contradiction. In the second case, $\bar{A}$ is a 2-transitive Frobenius group of degree $p+1$ and of order $p(p+1)$. Thus, $\bar{A}$ contains a regular elementary abelian normal subgroup $M$ with $|M|=p+1=q^{\ell}$. As $|\bar{A}|=p(p+1)$, we have that $M$ is a unique Sylow $q$-subgroup of $\bar{A}$. Since $\overline{\hat{D}_{2 n}} \leq \bar{A}$ and $\left|\hat{D}_{2 n}\right|=p+1$, we find that $\overline{\hat{D}_{2 n}}=M$ is normal in $\bar{A}$, implying that $\hat{D}_{2 n}$ is normal in $A$.

We may therefore assume that $N=\hat{C}_{n}$. Since $\hat{C}_{n}$ is normal in $A$, Proposition 6.2 gives us that $\hat{D}_{2 n}$ is normal in $A$.

To wrap up this paper, we mention a direct consequence of Theorem 1.1 to chiral dihedral maps.

A map $\mathcal{M}$ is an embedding of a finite connected graph $\Gamma$ into a surface so that it divides the surface into simply connected regions, called the faces of $\mathcal{M}$. To each face $f$, there is an associated closed walk of $\Gamma$ with edges surrounding $f$. An automorphism of $\mathcal{M}$ is an automorphism of $\Gamma$ which preserves the above closed walks of $\Gamma$. A map is regular in the sense of [26] (also called rotary) if it admits an automorphism cyclically permuting the consecutive edges of a face $f$ (as a one-step rotation of $f$ ) and an automorphism cyclically permuting the consecutive edges incident to some vertex $v$ of $f$ (as a one-step rotation of the neighbors of $v$ ). Hence, the automorphism group of a regular map acts transitively on the vertex set, arc set, and face set. If in 
addition, a regular map $\mathcal{M}$ contains an automorphism which "flips" an edge $e$ of a face $f$ and preserves $f$, we say that $\mathcal{M}$ is reflexible. If no such automorphism exists, then $\mathcal{M}$ is called chiral. In the latter case, $\operatorname{Aut}(\mathcal{M})$ acts regularly on the arc set of $\mathcal{M}$. A map $\mathcal{M}$ is said to be a Cayley map over a group $G$ if $\operatorname{Aut}(\mathcal{M})$ contains a subgroup isomorphic to $G$ which acts regularly on the vertex set of $\mathcal{M}$. In particular, a Cayley map over a dihedral group is also referred to as a dihedral map. Maps in general and dihedral maps in particular have recently been one main focus of research interest in the algebraic combinatorics community (see $[2,3,5,9,20,21]$ ).

We may now rephrase Theorem 1.1 in the context of dihedral maps.

Corollary 6.4 Let $\mathcal{M}$ be a chiral dihedral map with underlying graph $\Gamma$ such that $\operatorname{Aut}(\mathcal{M})=\operatorname{Aut}(\Gamma)$. Then the unique cyclic subgroup of index 2 in the corresponding regular dihedral subgroup $D$ of $\operatorname{Aut}(\mathcal{M})$ has nontrivial core in $\operatorname{Aut}(\mathcal{M})$.

Proof Since $\mathcal{M}$ is chiral, $\Gamma$ is not isomorphic to $K_{4}$; thus, $D \cong D_{2 n}$, where $n>2$, and $D$ contains a unique cyclic subgroup $C$ of index 2 . Clearly, $\Gamma$ is 1 -regular. Hence, if $C$ were core-free in $\operatorname{Aut}(\Gamma)$, then $\Gamma$ would belong to the family $\mathcal{F}$. But in view of Theorem 1.1, no graph in $\mathcal{F}$ is 1 -regular, a contradiction.

\section{References}

1. Biggs, N.L.: Algebraic Graph Theory, 2nd edn. Cambridge University Press, Cambridge (1993)

2. Conder, M., Jajcay, R., Tucker, T.: Regular $t$-balanced Cayley maps. J. Combin. Theory, Ser. B 97, 453-473 (2007)

3. Conder, M., Jajcay, R., Tucker, T.: Regular Cayley maps for finite abelian groups. J. Algebr. Comb. 25, 259-283 (2007)

4. Du, S.F., Malnič, A., Marušič, D.: Classification of 2-arc-transitive dihedrants. J. Combin. Theory, Ser. B 98, 1349-1372 (2008)

5. Feng, R., Wang, Y.: Regular balanced Cayley maps for cyclic, dihedral and generalized quaternion groups. Acta Math. Sinica (Engl. Ser.) 21, 773-778 (2005)

6. Herzog, M., Kaplan, G.: Large cyclic subgroups contain non-trivial normal subgroups. J. Group Theory 4, 247-253 (2001)

7. Kim, D., Kwon, Y.S., Lee, J.: Classification of $p$-valent regular Cayley maps on dihedral groups, manuscript

8. Kovács, I.: Classifying arc-transitive circulants. J. Algebr. Comb. 20, 353-358 (2004)

9. Kwak, Y.H., Kwon, Y.S., Feng, R.: Classification of $t$-balanced Cayley maps on dihedral groups. Eur. J. Comb. 27, 382-393 (2006)

10. Kwak, Y.H., Oh, Y.M.: One-regular normal Cayley graphs on dihedral groups of valency 4 or 6 with cyclic vertex stabilizer. Acta Math. Sin. (Engl. Ser.) 22, 1305-1320 (2006)

11. Kwak, Y.H., Kwon, Y.S., Oh, Y.-M.: Infinitely many one-regular Cayley graphs on dihedral groups of any prescribed valency. J. Comb. Theory, Ser. B 98, 585-598 (2008)

12. Li, C.H.: Permutation groups with a cyclic regular subgroup and arc-transitive circulants. J. Algebr. Comb. 21, 131-136 (2005)

13. Li, C.H.: Finite edge-transitive Cayley graphs and rotary Cayley maps. Trans. Am. Math. Soc. 358, 4605-4635 (2006)

14. Li, C.H., Finite, J. Pan: 2-arc transitive abelian Cayley graphs. Eur. J. Comb. 29, 148-158 (2007)

15. Marušič, D.: On 2-arc-transitivity of Cayley graphs. J. Comb. Theory, Ser. B 87, 162-196 (2003)

16. Marušič, D.: Corrigendum to "On 2-arc-transitivity of Cayley graphs". J. Comb. Theory, Ser. B 96, 761-764 (2006) [J. Comb. Theory, Ser. B 87, 162-196 (2003)]

17. McSorley, J.P.: Cyclic permutations in doubly-transitive groups. Commun. Algebra 25, 33-35 (1997)

18. Muzychuk, M.: On the structure of basic sets of Schur rings over cyclic groups. J. Algebra 169(2), 655-678 (1994) 
19. Muzychuk, M.: On the isomorphism problem for cyclic combinatorial objects. Discrete Math. 197/198, 589-605 (1999)

20. Nedela, R.: Regular maps-combinatorial objects relating different fields of mathematics. J. Korean Math. Soc. 38, 1069-1105 (2001)

21. Richter, B., Širán̆, J., Jajcay, R., Tucker, T., Watkins, M.: Cayley maps. J. Combin. Theory, Ser. B 95, 189-245 (2005)

22. Wang, C.Q., Xu, M.Y.: Non-normal one-regular and 4-valent Cayley graphs of dihedral groups $D_{2 n}$. Eur. J. Comb. 27, 750-766 (2006)

23. Wang, C.Q., Zhou, Z.Y.: One-regularity of 4-valent and normal Cayley graphs of dihedral groups $D_{2 n}$. Acta Math. Sin. (Chin. Ser.) 49, 669-678 (2006)

24. Wielandt, H.: Zur Theorie der einfach transitiven Permutationsgruppen II. Math. Z. 52, 384-393 (1949)

25. Wielandt, H.: Finite Permutation Groups. Academic Press, New York (1964)

26. Wilson, S.: Operators over regular maps. Pac. J. Math. 81, 559-568 (1979)

27. Xu, M.Y.: Automorphism groups and isomorphisms of Cayley digraphs. Discrete Math. 182, 309-319 (1998) 